\title{
Supporting Information: Reverse Protonation of Buried Ion-pairs in Staphylococcal Nuclease
}

\section{Mutants}

\author{
Jiahua Deng ${ }^{\dagger}$ and Qiang Cui ${ }^{*, \ddagger}$ \\ $\dagger$ Department of Chemistry, Boston University, 590 Commonwealth Avenue Boston, MA \\ 02215 \\ $\ddagger$ Departments of Chemistry, Physics and Biomedical Engineering, Boston University, 590 \\ Commonwealth Avenue Boston, MA 02215 \\ E-mail: qiangcui@bu.edu, Tel:(+1)-617-353-6189
}




\section{Charging free energy calculations}

Table S1: Components (in kcal/mol) from charging free energy simulations. ${ }^{a}$

\begin{tabular}{lccccr}
\hline Component & EK & $\mathrm{KE}$ & $\mathrm{EK}_{\text {neutral }}$ & $\mathrm{KE}_{\text {neutral }}$ & $\Delta+\mathrm{PHS}$ \\
\hline$\Delta G_{Q}^{U}$ & $-134.3 \pm 0.1$ & $-134.3 \pm 0.1$ & $-37.5 \pm 0.04$ & $-37.5 \pm 0.04$ & $-6.6 \pm 0.01$ \\
\hline$\Delta G_{Q}^{F}$ & $-78.6 \pm 1.2$ & $-75.7 \pm 1.0$ & $-30.8 \pm 0.6$ & $-29.7 \pm 0.1$ & $-6.7 \pm 0.03$ \\
\hline
\end{tabular}

a. The decharging free energies $\left(\Delta G_{d Q}\right)$ cited in thermodynamic cycles shown in Figs. 5-6 in the main text are the opposite of corresponding charging free energies $\left(\Delta G_{Q}\right)$. See Tables S2-S3 for additional analyses.

Table S2: Charging free energy of individual blocked amino acid sidechains in solution (in $\mathrm{kcal} / \mathrm{mol}$ ) for the analysis of unfolded state. ${ }^{a}$

\begin{tabular}{ccccccc}
\hline$\Delta \mathrm{G}_{Q}^{U}$ & Glu & Lys & Val & Leu & GluP & Lsn \\
\hline intra & -12.0 & 12.7 & -2.6 & -11.4 & -35.4 & 1.9 \\
\hline inter & -77.9 & -57.1 & 4.6 & 2.8 & -2.4 & -1.6 \\
\hline total & $-89.9 \pm 0.08$ & $-44.4 \pm 0.06$ & $2.0 \pm 0.01$ & $-8.6 \pm 0.01$ & $-37.8 \pm 0.02$ & $0.23 \pm 0.04$ \\
\hline
\end{tabular}

a. Blocked sidechain analogs solvated in a TIP3P water droplet with a radius of $25 \AA$. "intra" refer to contributions from within the sidechain, and "inter" refer to contributions from sidechain-environment interactions. b. GluP: protonated Glu; Lsn: neutral Lys.

Table S3: Components of charging free energy of folded states (in $\mathrm{kcal} / \mathrm{mol}$ ) relative to solution. ${ }^{a}$

\begin{tabular}{cccccrrr}
\hline$\Delta \mathrm{G}_{Q}^{F}$ & $\mathrm{EK}$ & $\mathrm{KE}$ & $\mathrm{EK}_{\text {neutral }}$ & $\mathrm{KE}_{\text {neutral }}$ & $\Delta+\mathrm{PHS}$ & $\mathrm{E}^{-} \cdot \mathrm{KH}^{+}{ }_{a q}$ & $\mathrm{EH} \cdot \mathrm{K}_{a q}$ \\
\hline intra & -47.3 & -51.1 & -34.5 & -33.3 & -13.8 & 0.7 & -33.5 \\
\hline inter & -31.3 & -24.6 & 3.7 & 3.6 & 7.1 & -135.0 & -4.0 \\
\hline total & $-78.6 \pm 1.2$ & $-75.7 \pm 1.0$ & $-30.8 \pm 0.6$ & $-29.7 \pm 0.1$ & $-6.7 \pm 0.03$ & $-134.3 \pm 0.1$ & $-37.5 \pm 0.04$ \\
\hline \multicolumn{7}{c}{ a. See footnote a of Table S2 for the definition of "intra" and "inter" contributions. }
\end{tabular}

Table S4: Van der Waals interaction energies (in kcal/mol) between the perturbed sidechains and the environment in de-charged windows. ${ }^{a}$

\begin{tabular}{lccccr}
\hline VdW & EK & EK $_{\text {neutral }}$ & KE & KE $_{\text {neutral }}$ & $\Delta+\mathrm{PHS}$ \\
\hline interaction energy $(\lambda=0.0)$ & $-21.4 \pm 0.09$ & $-21.3 \pm 0.3$ & $-21.5 \pm 0.07$ & $-22.4 \pm 0.1$ & $-16.9 \pm 0.06$ \\
\hline
\end{tabular}

a. While the van der Waals contribution to the mutation free energies in the folded states $\left(\Delta G_{m u t}^{F^{0}}\right.$ in Fig. 5 and $\Delta G_{m u t}^{F(C / N)^{0}}$ in Fig. 6 in the main text) can, in principle, be evaluated using free energy perturbation,

the interaction energies computed here strongly support that the contribution is small $(<1 \mathrm{kcal} / \mathrm{mol})$, especially for EK vs. KE in either ionization state. For the difference between Glu-Lys and Val-Leu (i.e., $\mathrm{EK} / \mathrm{KE}$ or $\mathrm{EK}_{\text {neutral }} / \mathrm{KE}_{\text {neutral }}$ vs. $\left.\Delta+\mathrm{PHS}\right)$, the difference is larger and $\sim 4 \mathrm{kcal} / \mathrm{mol}$. As indicated in the footnote of Table 2 in the main text, including this contribution will in fact bring the computed relative folding stability between $\mathrm{EK}_{\text {neutral }} / \mathrm{KE}_{\text {neutral }}$ and $\Delta+\mathrm{PHS}$ closer to the experimental value, but represents a negligible impact on the folding stability difference between EK/KE and $\Delta+\mathrm{PHS}$. 


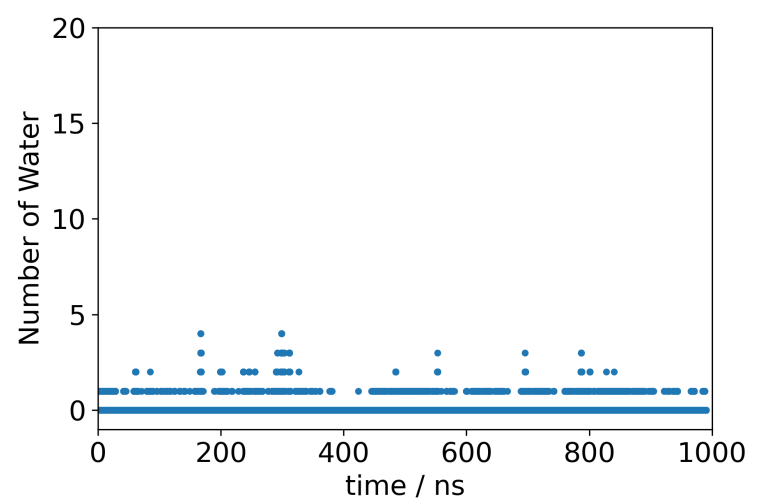

(a)

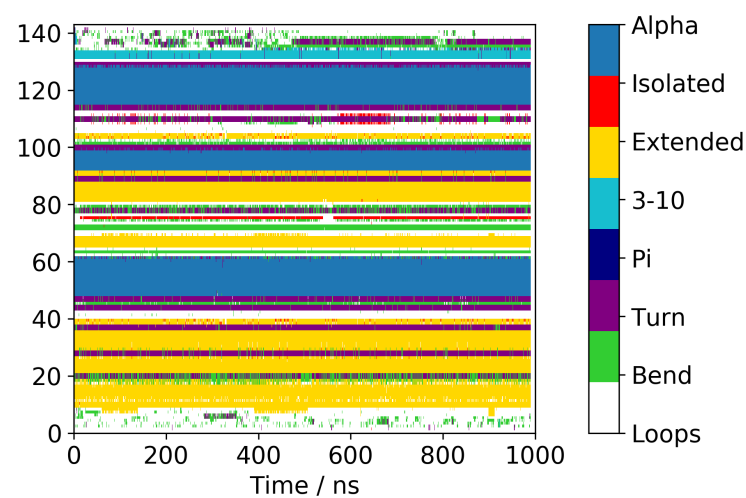

(b)

Figure S1: The $\Delta+$ PHS background features (a) low hydration (only one water molecule in the crystal structure) and (b) stable secondary structures during the MD simulations.

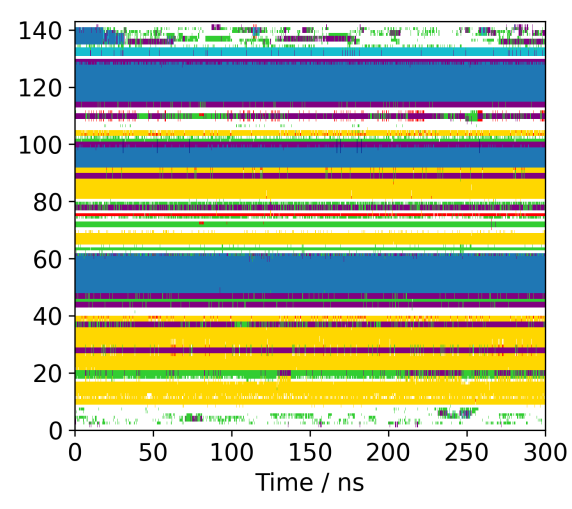

(a)
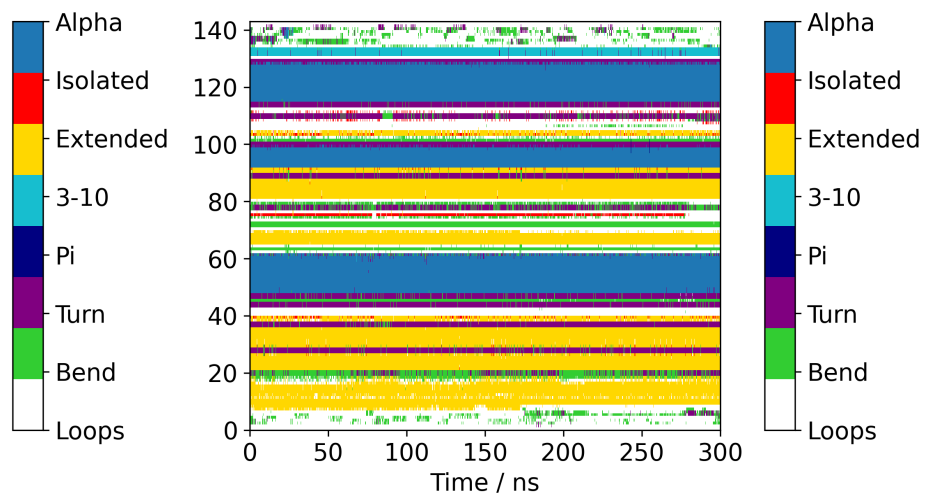

(b)

Figure S2: Secondary structure evolution during the MD simulation for the (a) $\mathrm{EK}_{\text {neutral }}$ and (b) $\mathrm{KE}_{\text {neutral }}$ simulations. 


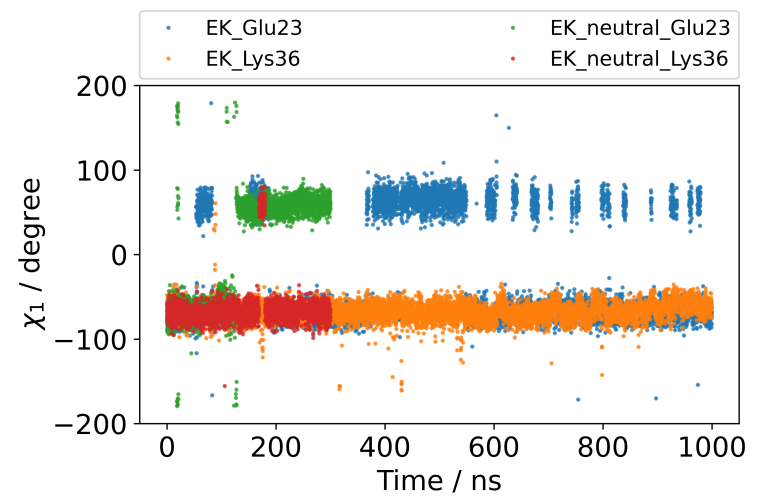

(a)

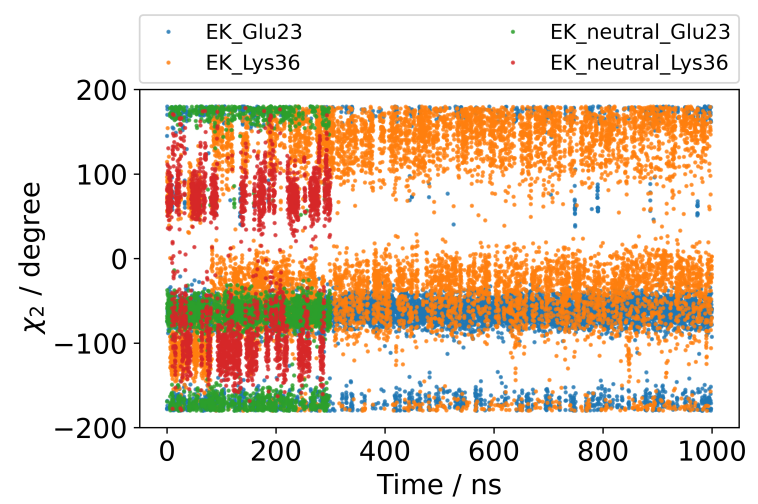

(c)

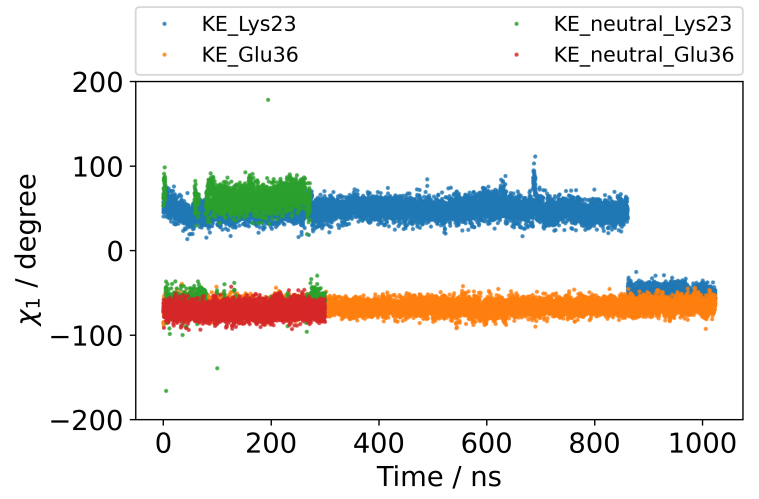

(b)

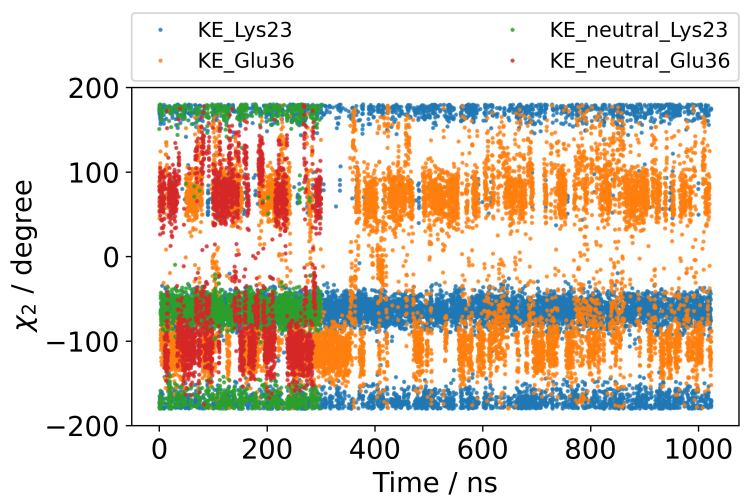

(d)

Figure S3: $\chi_{1}$ and $\chi_{2}$ of residues 23 and 36 in the EK, $\mathrm{EK}_{\text {neutral }}$, KE and $\mathrm{KE}_{\text {neutral }}$ simulations.

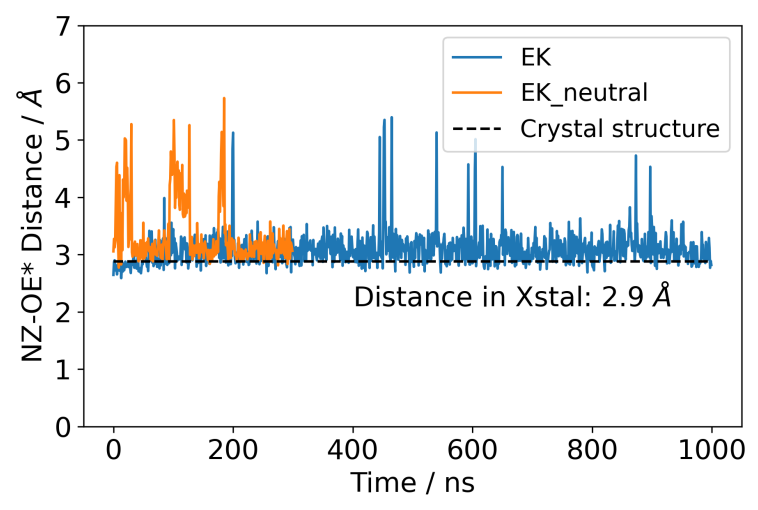

(a)

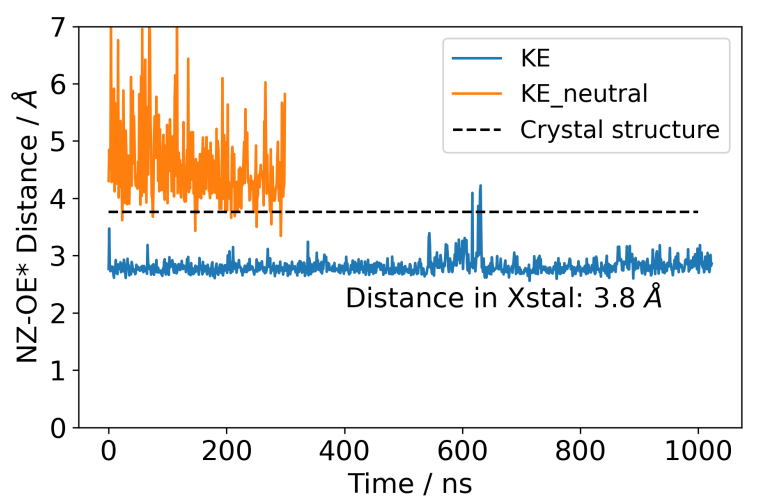

(b)

Figure S4: The distance between Glu and Lys in the (a) EK/EK $\mathrm{E}_{\text {neutral }}$ and (b) $\mathrm{KE} / \mathrm{KE}_{\text {neutral }}$ simulations. The distances are averaged over NZ-OE1 and NZ-OE2 distances, respectively. Black dashed lines indicate the corresponding distances in the EK and KE crystal structures, respectively. 


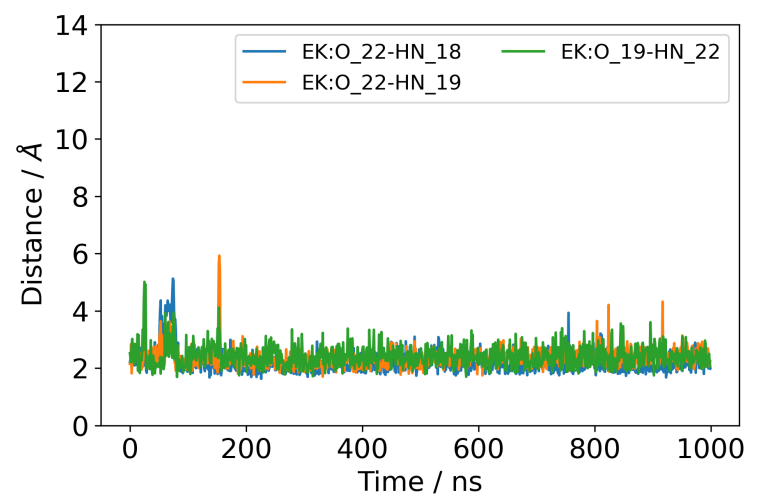

(a)

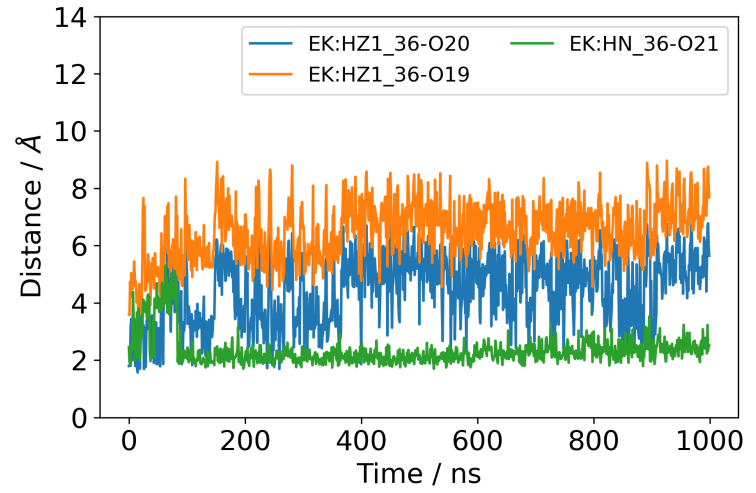

(c)

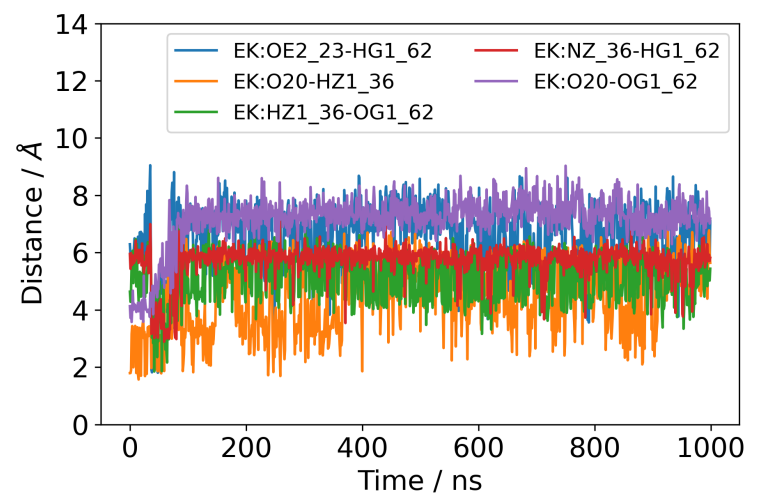

(e)

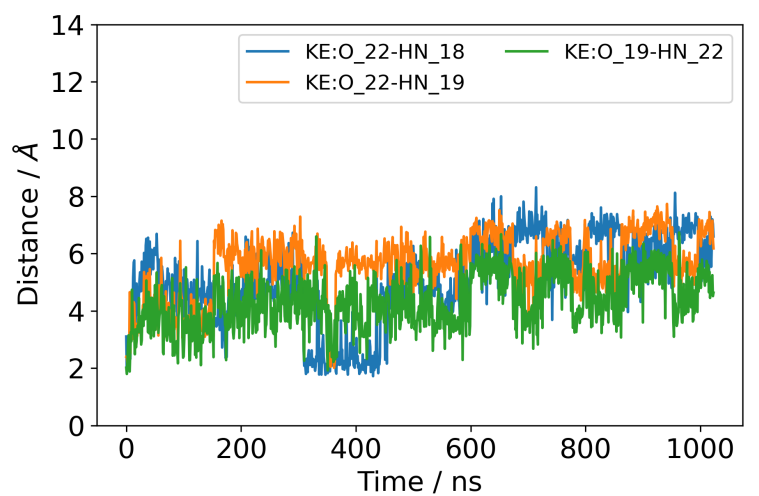

(b)

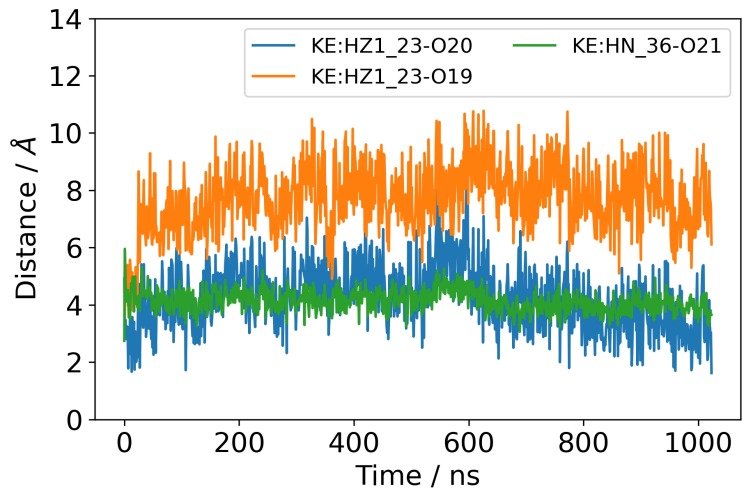

(d)

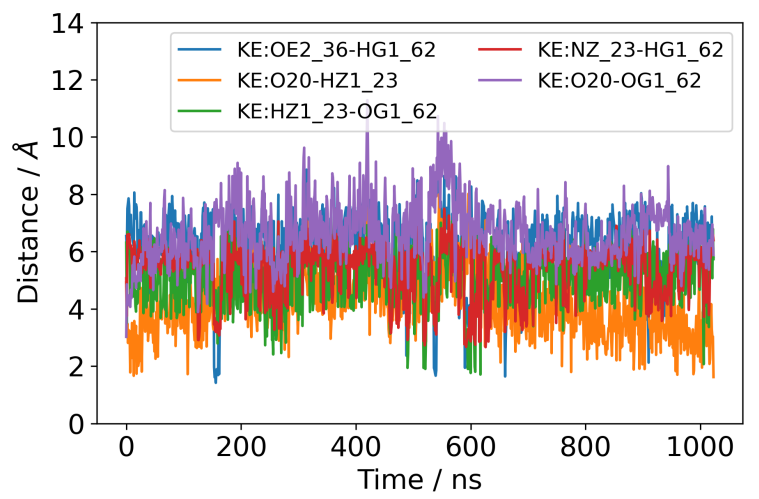

(f)

Figure S5: Distances of potential hydrogen bonds during molecular dynamics for the EK (left) and KE (right) simulations. (a-b) Hydrogen bonds between $\beta 1$ and $\beta 2$ strands; (c-d) hydrogen bonds between Glu/Lys and nearby residues; (e-f) hydrogen bonds observed in crystal structures exhibit large fluctuations during simulations. 


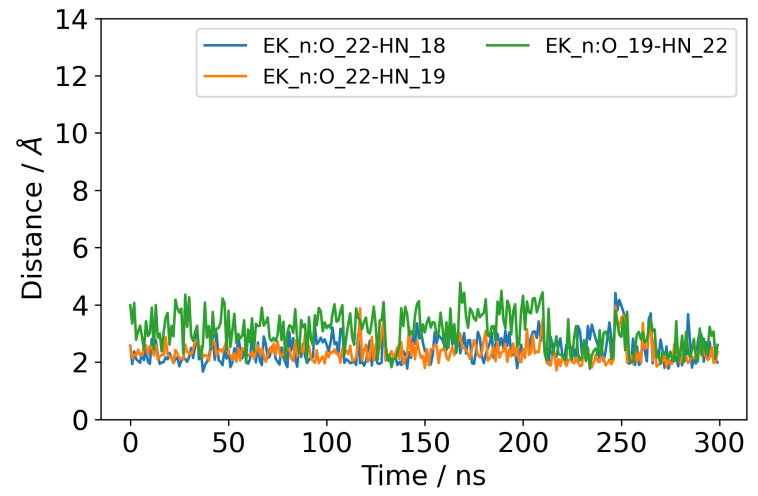

(a)

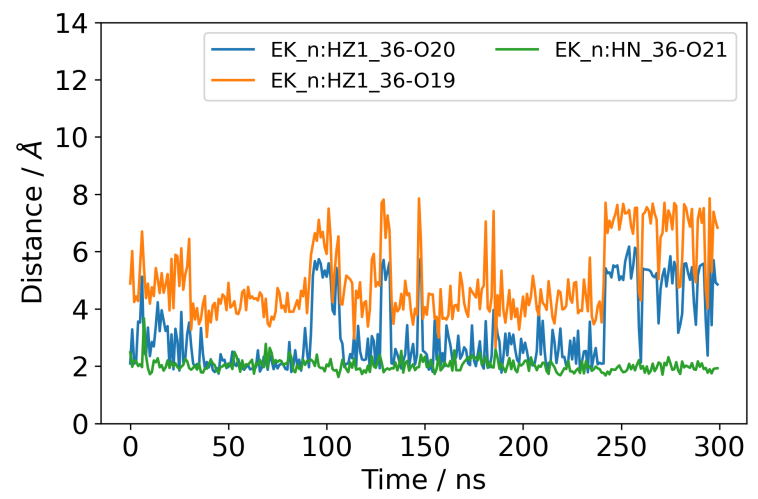

(c)

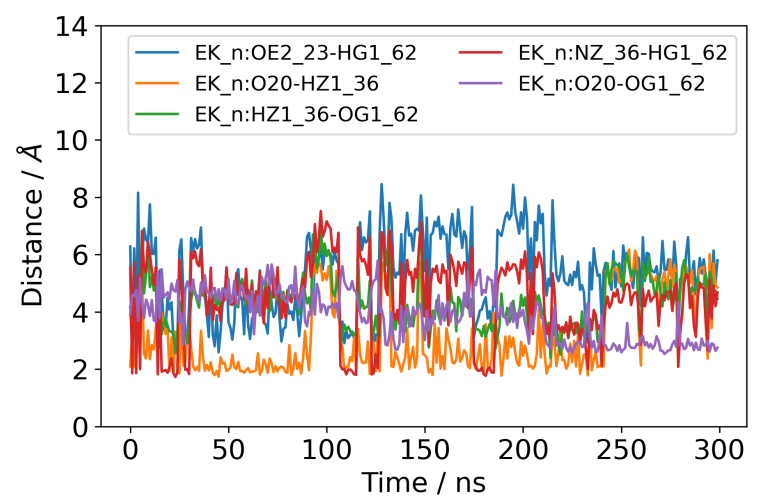

(e)

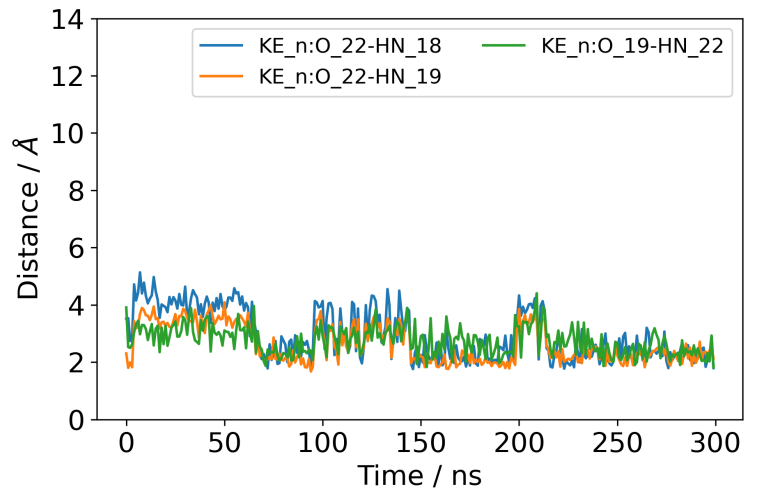

(b)

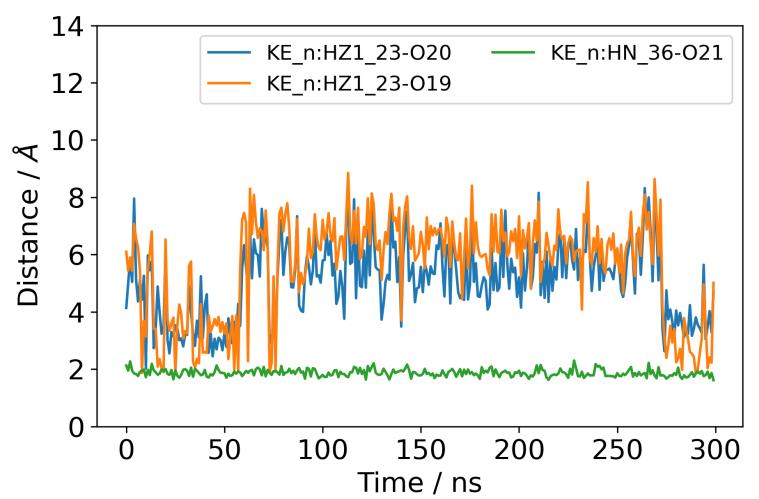

(d)

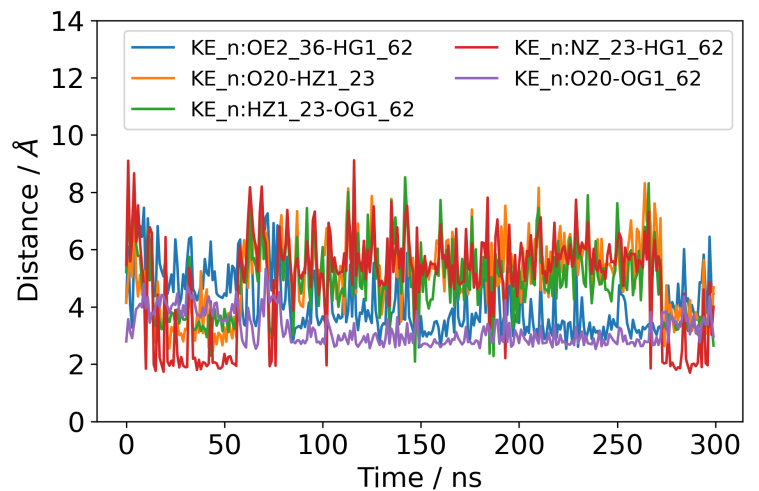

(f)

Figure S6: Same as Fig. S5, but for the $\mathrm{EK}_{\text {neutral }}$ (left) and $\mathrm{KE}_{\text {neutral }}$ (right) simulations. 


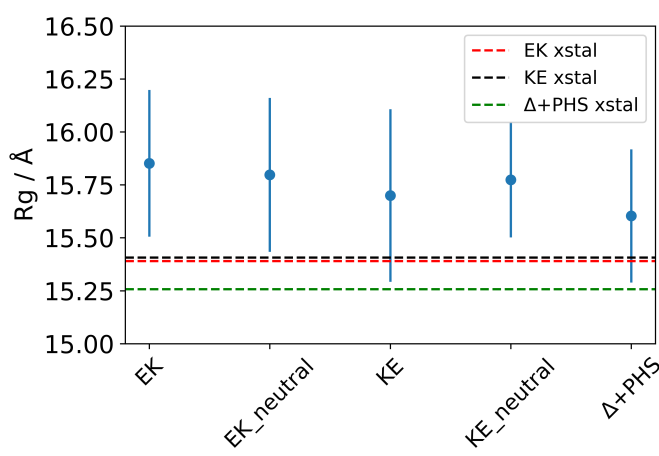

(a)

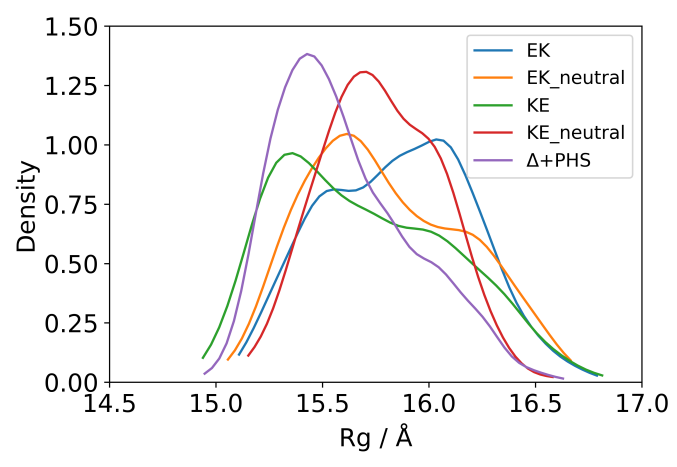

(b)

Figure S7: Radius of gyration ( $R_{g}$ in Angstrom) during MD simulations of the SNase variants. (a) Average values of $R_{g}$; error bars represent the corresponding standard deviations. Red, black, and green dashed lines indicate the $R_{g}$ values in the EK, KE, and $\Delta+\mathrm{PHS}$ crystal structures, respectively. (b) Density estimates of $R_{g}$ using a Gaussian kernel for each system. For all systems studied, no over-compact structures compared to the crystal structures are observed. 


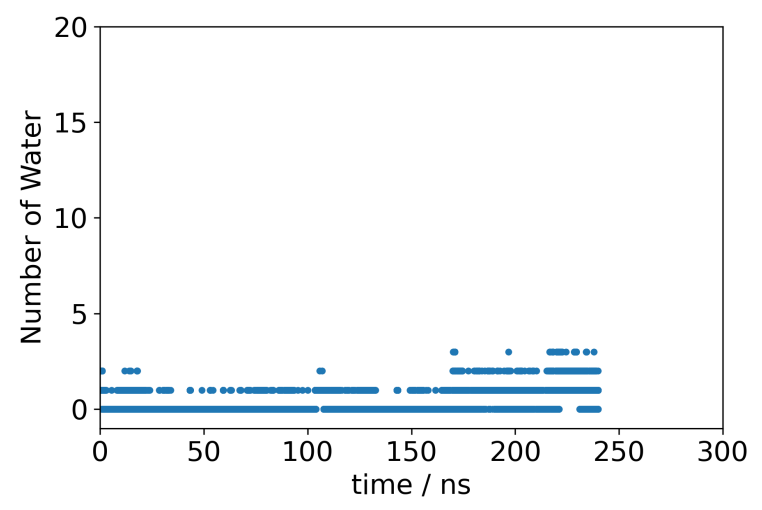

(a)

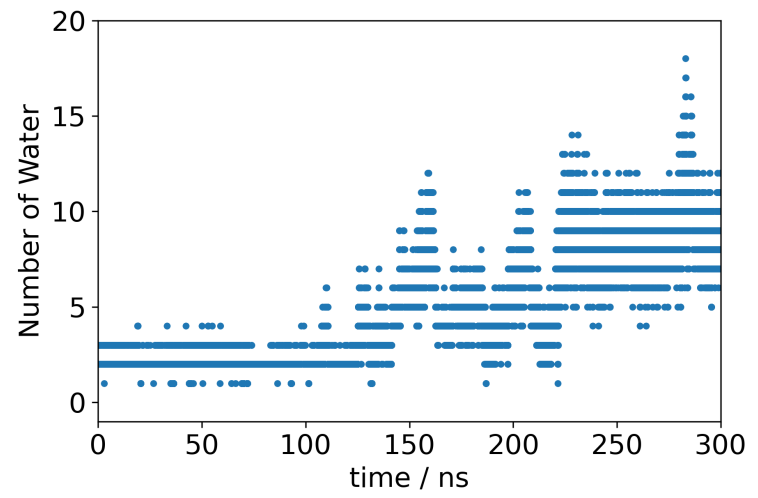

(c)

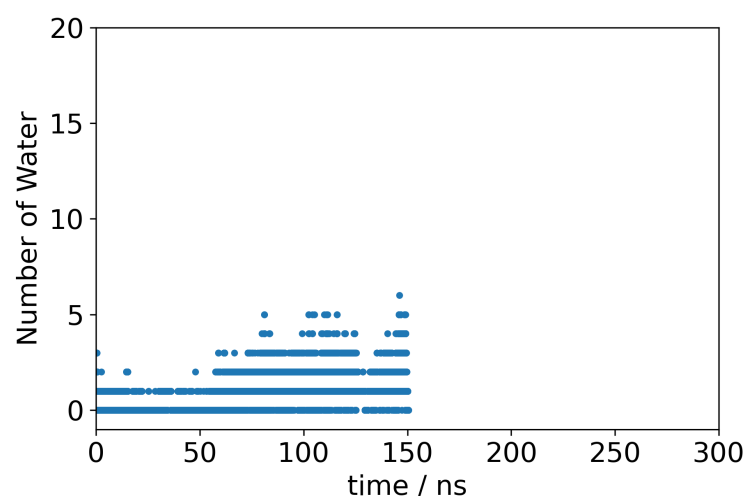

(b)

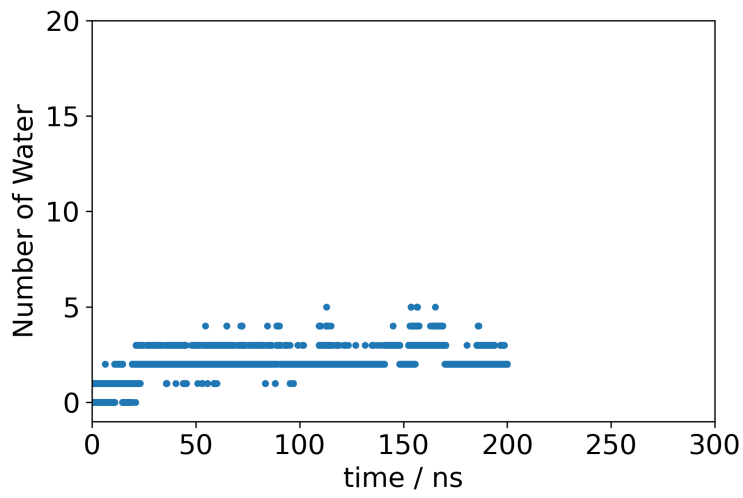

(d)

Figure S8: Hydration level for intermediate- $\lambda$ windows takes a long time to equilibrate. Number of cavity water in (a) $\lambda=0.2$ window, (b) $\lambda=0.2$ window starting from a snapshot at 120 ns from the $\lambda=0.8$ window, (c) $\lambda=0.8$ window, and (d) $\lambda=0.8$ window starting from a snapshot at $120 \mathrm{~ns}$ from $\lambda=0.2$ window. 


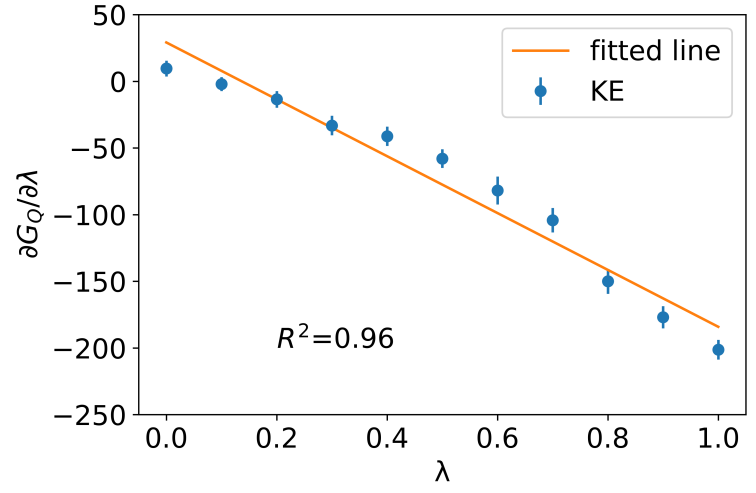

(a)

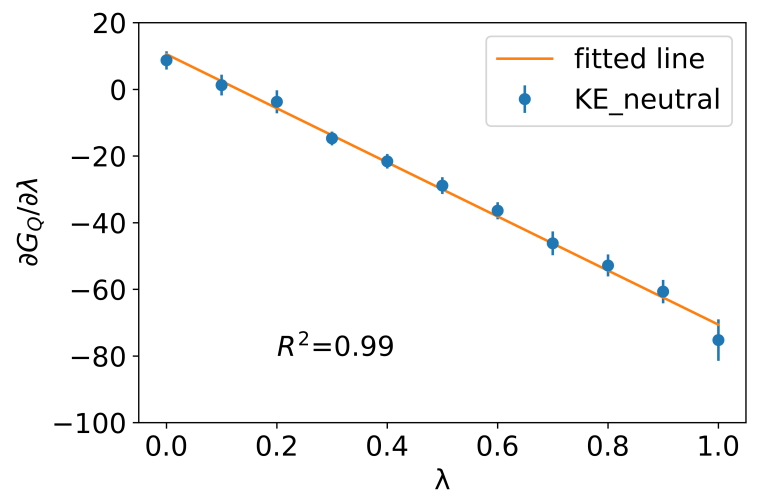

(c)

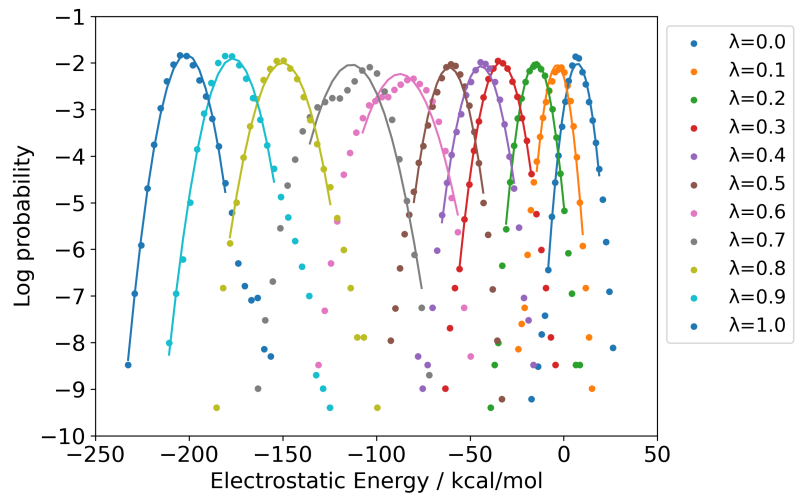

(b)

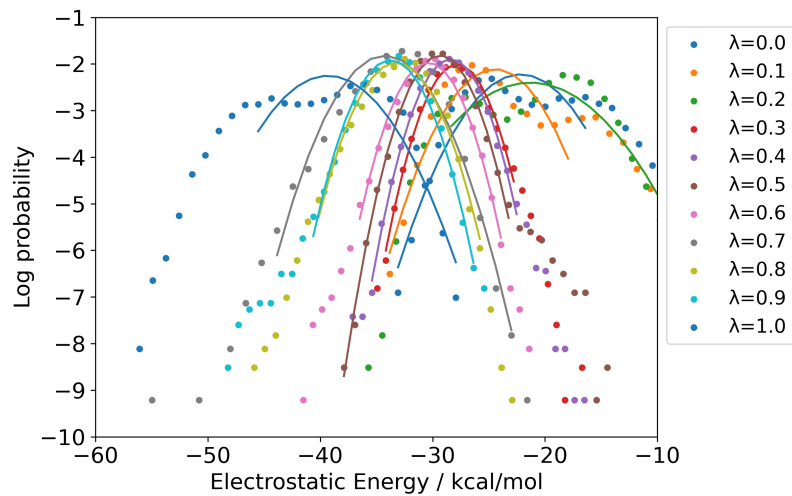

(d)

Figure S9: Same as Fig. 4 of the main text, except that results for the KE (top) and $\mathrm{KE}_{\text {neutral }}$ (bottom) variants are shown. 


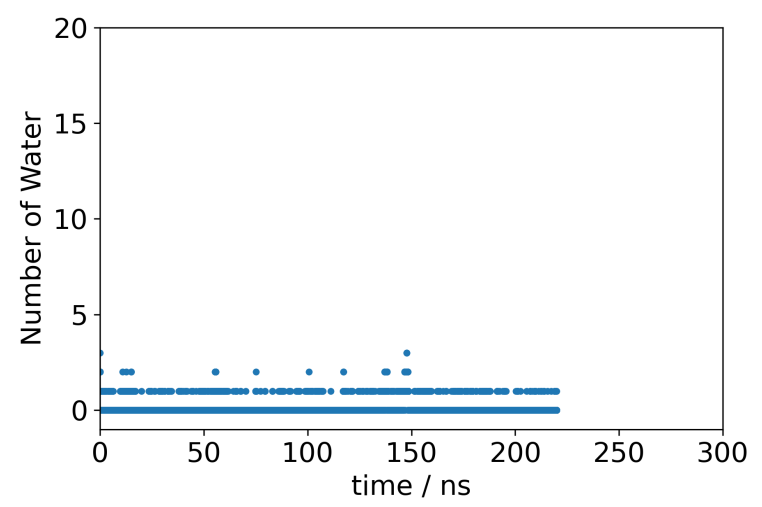

(a)

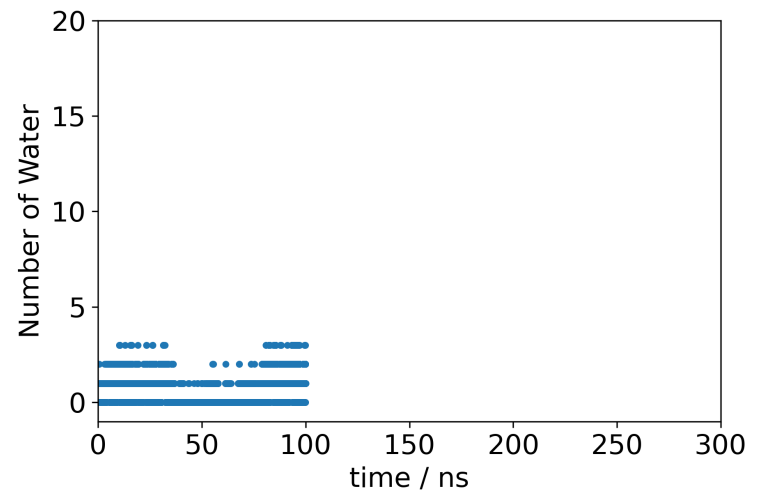

(c)

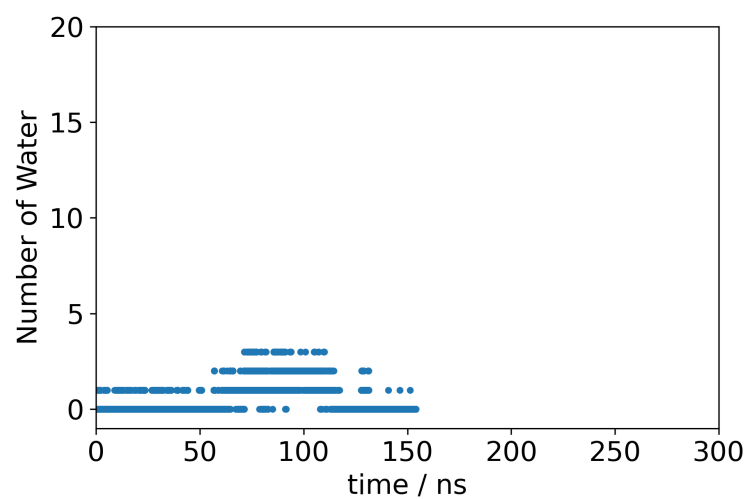

(b)

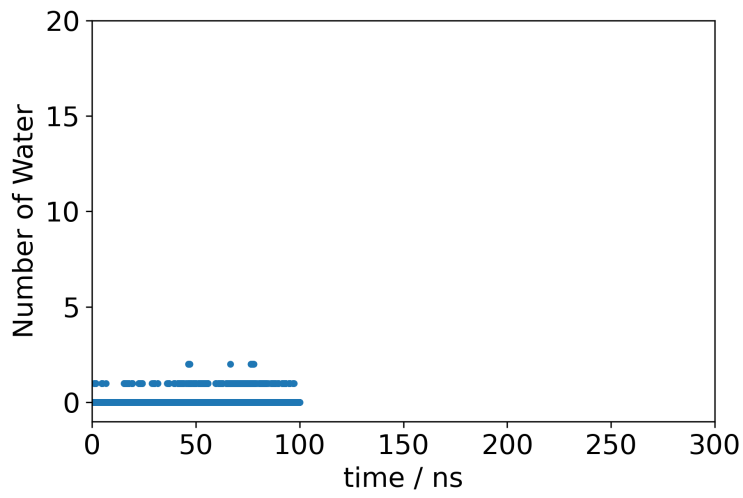

(d)

Figure S10: The hydration level of the de-charged states of (a) EK, (b) KE, (c) EK neutral $_{\text {, }}$ and (d) $\mathrm{KE}_{\text {neutral }}$ in free energy simulations are similar to each other and close to $\Delta+\mathrm{PHS}$ (see Figure S1a). This similarity justifies treating all de-charged states being identical in the thermodynamical cycles shown in Figs. 5 and 6 in the main text. 


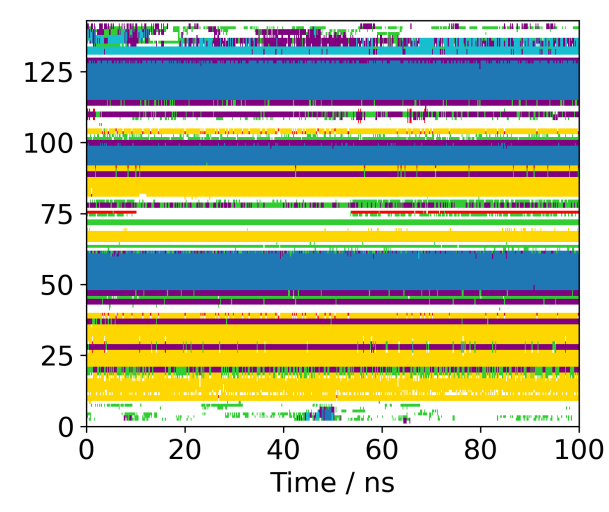

(a)

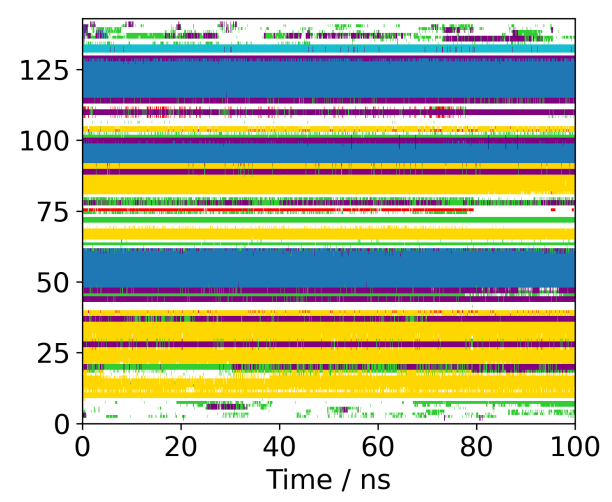

(c)

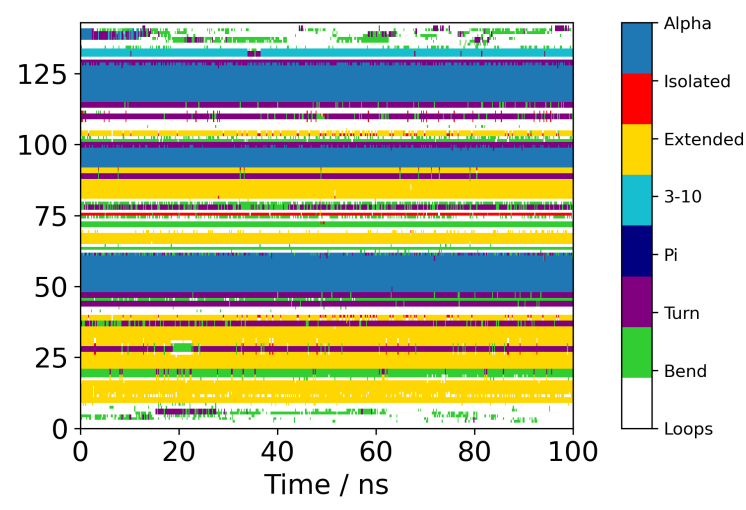

(b)

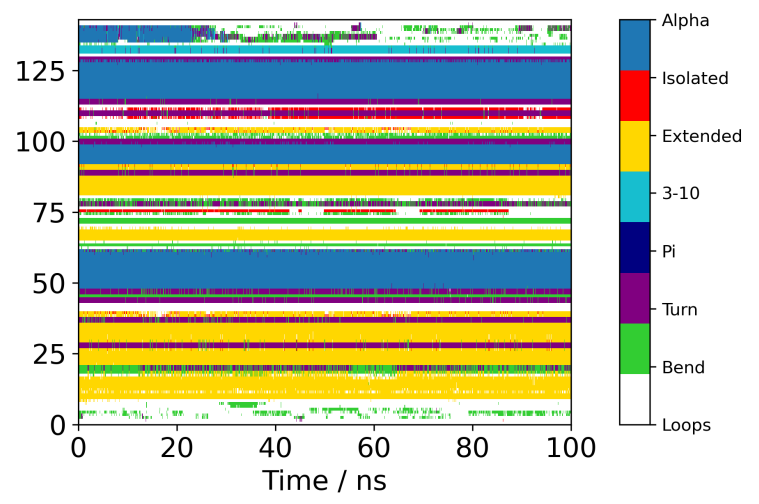

(d)

Figure S11: Secondary structure of the de-charged states of (a) EK, (b) KE, (c) EK neutral, and (d) $\mathrm{KE}_{\text {neutral }}$ also remain similar to each other and close to $\Delta+\mathrm{PHS}$ (see Figure S1b) during the MD simulation; this observation further justifies treating all de-charged states being identical in the thermodynamical cycles shown in Figs. 5 and 6 in the main text. 